\title{
Functional genomic screen for modulators of ciliogenesis and cilium length
}

\author{
Joon Kim ${ }^{1}$, Ji Eun Lee ${ }^{1}$, Susanne Heynen-Genel ${ }^{2}$, Eigo Suyama ${ }^{2}$, Keiichiro Ono ${ }^{3}$, KiYoung Lee ${ }^{3,4}$, Trey Ideker $^{3}$, \\ Pedro Aza-Blanc ${ }^{2} \&$ Joseph G. Gleeson ${ }^{1}$
}

Primary cilia are evolutionarily conserved cellular organelles that organize diverse signalling pathways ${ }^{1,2}$. Defects in the formation or function of primary cilia are associated with a spectrum of human diseases and developmental abnormalities ${ }^{3}$. Genetic screens in model organisms have discovered core machineries of cilium assembly and maintenance ${ }^{4}$. However, regulatory molecules that coordinate the biogenesis of primary cilia with other cellular processes, including cytoskeletal organization, vesicle trafficking and cell-cell adhesion, remain to be identified. Here we report the results of a functional genomic screen using RNA interference (RNAi) to identify human genes involved in ciliogenesis control. The screen identified 36 positive and 13 negative ciliogenesis modulators, which include molecules involved in actin dynamics and vesicle trafficking. Further investigation demonstrated that blocking actin assembly facilitates ciliogenesis by stabilizing the pericentrosomal preciliary compartment (PPC), a previously uncharacterized compact vesiculotubular structure storing transmembrane proteins destined for cilia during the early phase of ciliogenesis. The PPC was labelled by recycling endosome markers. Moreover, knockdown of modulators that are involved in the endocytic recycling pathway affected the formation of the PPC as well as ciliogenesis. Our results uncover a critical regulatory step that couples actin dynamics and endocytic recycling with ciliogenesis, and also provides potential target molecules for future study.

Cilium assembly and disassembly are interconnected with several complex cellular processes such as cell cycle, cell polarization and cell migration, suggesting that there may be large numbers of ciliogenesis modulators ${ }^{1,5}$. In accordance with this, recent proteomic analyses together with comparative genomics and bioinformatics studies have identified over a thousand cilia- or basal body-associated proteins, referred to as the "ciliome"6,7. Although these approaches can provide a comprehensive list of candidates, the discovery of key regulators of ciliogenesis, which could reveal potential therapeutic targets, requires functional analysis. Thus we developed a high-throughput assay using small interfering RNA (siRNA) to evaluate the functional impact of 7,784 therapeutically relevant genes across the human genome (Supplementary Table 1). The screen was based on an in vitro ciliogenesis model: serum starvation-induced ciliogenesis in telomerase-immortalized human retinal pigmented epithelial (htRPE) cells. Enhanced green fluorescent protein (EGFP)-tagged Smoothed (Smo), a transmembrane protein that accumulates in primary cilia ${ }^{8}$, was used as a cilium marker for automated quantification of ciliated cells (Supplementary Fig. $1 \mathrm{a}-\mathrm{c}$ ). Sensitivity of the screen strategy was assessed by siRNAs targeting KIF3A, a critical protein for cilium assembly (Supplementary Fig. 1d). We selected a screen condition suitable for identifying both positive and negative modulators: siRNA transfection was performed at a reduced (suboptimal) cell density to detect these dynamic ranges of ciliogenesis activity, and ciliation was assessed after $48 \mathrm{~h}$ of serum starvation (Supplementary Fig. 1e, f).

The primary screen identified 153 positive modulator hits and 79 negative modulator hits (Supplementary Table 1 and Supplementary Fig. 2a). We next performed a confirmation screen with the following modifications: (1) optimal cell density for ciliogenesis (confluent at the time of serum starvation) was used to minimize the selection of siRNAs that influence ciliogenesis indirectly through an effect on cell density, (2) cilium length was measured for validating negative modulators because length increase was a highly specific indicator for enhanced ciliogenesis, where optimal ciliogenesis condition was used (Supplementary Fig. 2b). The confirmation screen identified 40 positive and 13 negative modulators, which are associated with various molecular processes (Supplementary Table 2 and Supplementary Fig. 3). Notably, the screen hits include INPP5E, a gene recently identified as mutated in a human ciliopathy, Joubert syndrome 9 . A positive modulator Agtpbp1 (formerly Nna1) is mutated in Purkinje cell degeneration ( $p c d$ ) mice, which exhibit ciliopathy phenotypes: retinal degeneration and defective spermatogenesis ${ }^{10}$. Although the library was selected for therapeutically relevant genes and thus not highly representative of ciliome genes (tending to be structural), we did confirm 12 genes encoding ciliome proteins (Supplementary Table 3 and Supplementary Fig. 3).

We rescreened the confirmed positive modulators using antiacetylated tubulin immunofluorescence as a cilium marker, and found four genes that did not affect cilium assembly (Supplementary Table 3 and Supplementary Fig. 3). The confirmed negative modulators were further classified according to silencing phenotypes observed in the absence of serum starvation. Silencing of five genes facilitated ciliogenesis even without serum starvation (Supplementary Table 3 and Supplementary Fig. 3).

Among the ciliogenesis modulators with high confirmation screen scores (Supplementary Table 2) are two gelsolin (GSN) family proteins GSN and AVIL, which regulate cytoskeletal actin organization by severing actin filaments ${ }^{11}$. Depletion of GSN proteins by two independent siRNAs significantly reduced ciliated cell numbers, indicating that actin filament severing is involved in ciliogenesis (Fig. 1a-c). In contrast, silencing of actin-related protein ACTR3 (also called ARP3), which is a major constituent of the ARP2/3 complex that is necessary for nucleating actin polymerization at filament branches ${ }^{12}$, caused a significant increase in cilium length (Fig. 1a, d, e) and also facilitated ciliogenesis independently of serum starvation (Fig. 1f). These observations indicate an inhibitory role of branched actin network formation in ciliogenesis. 

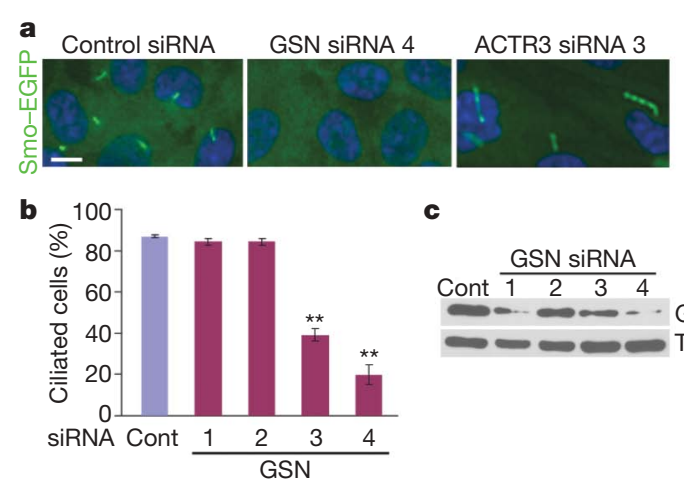

c

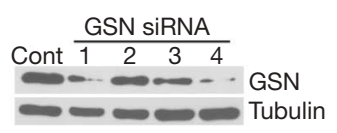

\section{i}

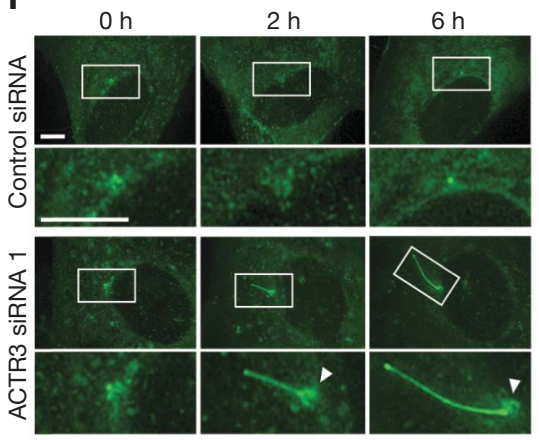

d

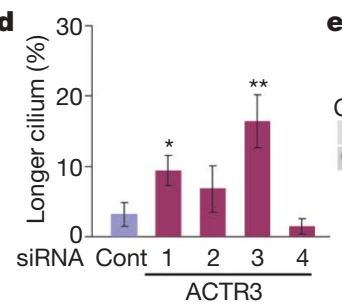

$\mathbf{g}$
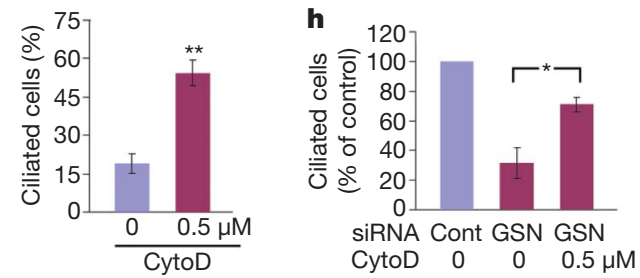

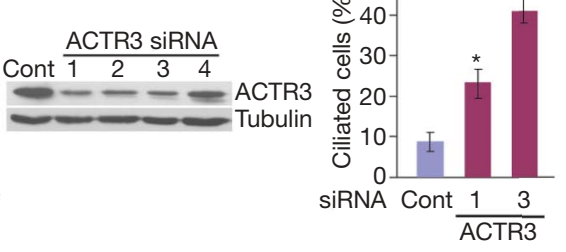

|

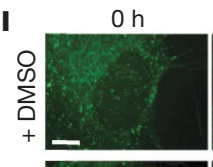

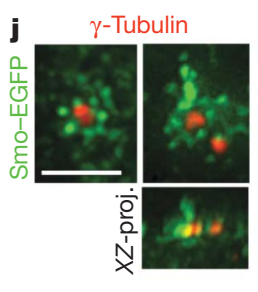

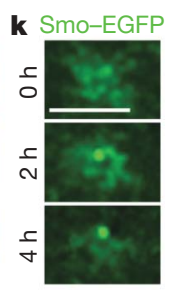

$2 \mathrm{~h}$

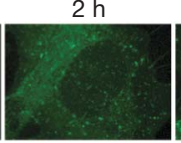

$6 \mathrm{~h}$

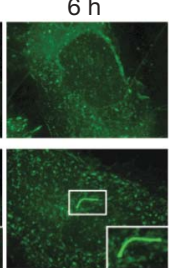

Figure 1 | Regulators of actin dynamics modulate cilium assembly. a, b, GSN knockdown reduced ciliated cell numbers. a, d, ACTR3 knockdown increased the number of cells with longer cilium $(>6 \mu \mathrm{m})$. c, e, Western blots showing protein levels (note that GSN siRNA 1 (c) was cytotoxic). f, ACTR3 knockdown induced ciliogenesis without serum starvation. g, Cytochalasin D treatment for $8 \mathrm{~h}$ increased ciliated cell numbers without serum starvation. $\mathbf{h}$, Ciliogenesis defect by GSN

knockdown was rescued by $8 \mathrm{~h}$ cytochalasin $\mathrm{D}$ treatment. i, Live imaging of

To examine the link between actin dynamics and ciliogenesis, we treated htRPE cells with actin polymerization inhibitor cytochalasin $\mathrm{D}$, which facilitated ciliogenesis independently of serum starvation and promoted cilium elongation (Fig. $1 \mathrm{~g}$ and Supplementary Fig. 4). The ciliogenic effect of cytochalasin D was also observed in HEK293T cells (Supplementary Fig. 5a, b). Moreover, cytochalasin D treatment significantly rescued the ciliogenesis defect caused by GSN knockdown (Fig. 1h). Cytochalasin D facilitated ciliogenesis at doses below that which affects stress fibre formation, excluding the possibility of global actin cytoskeleton rearrangement in ciliogenesis control (Supplementary Fig. 5c). Involvement of actin dynamics in ciliogenesis is further supported by the finding that $\alpha$-PARVIN (PARVA), a component of the focal adhesion complex regulating actin cytoskeletal dynamics and cell signalling ${ }^{13}$, exhibits knockdown phenotypes similar to that of ACTR3 (Supplementary Fig. 6).

To identify the mechanism of altered ciliogenesis, we performed time-course live imaging. Notably, a number of non-ciliated htRPE cells transfected with ACTR3 siRNAs (14/30 cells) developed pronounced primary cilia $(2.9 \pm 0.9 \mu \mathrm{m}$ in length) within $2 \mathrm{~h}$ of serum starvation, whereas none of the control cells ( $0 / 30$ cells) displayed cilia longer than $1.5 \mu \mathrm{m}$ (Fig. 1i and Supplementary Fig. 7a). Furthermore, faster cilium elongation after the initiation of ciliogenesis was observed in ACTR3-depleted cells (mean cilium extension rate for the first $6 \mathrm{~h}$ after serum starvation: ACTR3 depleted cells, $0.75 \pm 0.27 \mu \mathrm{mh}^{-1}$ $(n=17)$; control cells, $\left.0.11 \pm 0.06 \mu \mathrm{m} \mathrm{h}^{-1}(n=6)\right)$. These observations indicate that slow progression of ciliogenesis in htRPE cells is not the result of slow transduction of serum starvation-mediated ciliogenic signal or unavailability of core cilium assembly machineries, but is ascribed to the presence of an inhibitory regulation involving ACTR3.

htRPE-Smo-EGFP cells after $60 \mathrm{~h}$ siRNA transfection. Arrowheads, the pericentrosomal preciliary compartment (PPC) at the ciliary base. j, Morphology of PPC. Gamma-tubulin labels the centrosome. k, Live imaging of a PPC-positive cell in serum-free medium. I, Live cell imaging in serum-free medium containing dimethylsulphoxide (DMSO) or cytochalasin D. Values, mean \pm s.d. $(n=4(\mathbf{b}, \mathbf{d}), 2(\mathbf{f})$ and $3(\mathrm{~g}, \mathbf{h}))$. Cont, control; CytoD, cytochalasin D. Student's $t$-test: *, $P<0.05$; **, $P<0.01$. Scale bars, $10 \mu \mathrm{m}(\mathbf{a}) ; 5 \mu \mathrm{m}(\mathbf{i}, \mathbf{I}) ; 2.5 \mu \mathrm{m}(\mathbf{j}, \mathbf{k})$.

Earlier electron microscopic studies found small vesicles tightly associated with the centriole initiating cilium assembly in fibroblasts and smooth muscle cells $s^{14,15}$. This indicates that ciliogenesis in nonpolarized cells could be initiated by vesicle docking to the basal body, whereas basal body docking to the apical plasma membrane initiates ciliogenesis in polarized epithelial cells. More recent studies have also reported that the biogenesis of ciliary membrane that is coordinated with axoneme assembly may involve fusion of transport vesicles at the base of cilia ${ }^{16,17}$. Smo-EGFP allowed us to visualize transport vesicles targeted to the ciliary base. Interestingly, a subset of htRPE-SmoEGFP cells ( 10\%: 42/434 interphase cells) cultured in the presence of serum exhibited compact Smo-EGFP-positive vesiculotubular structures (termed here PPCs) tightly associated with the centrosome (Fig. 1j). In these cells, ciliogenesis occurred at the PPC during $4 \mathrm{~h}$ live imaging (Fig. 1k). Notably, an attenuation of Smo-EGFP-positive PPC was observed after the initiation of ciliogenesis (Fig. 2k and Supplementary Fig. $7 \mathrm{~b}$, control cells). After $4 \mathrm{~h}$ of serum starvation (when cilia are detected in $\sim 20 \%$ of cells), $3.4 \%$ of total cilia (17/500 cilia) were accompanied by PPCs, indicating that the transition of the PPC to cilia occurs over the course of hours. PPC-positive cilia were rarely observed after $24 \mathrm{~h}$ serum starvation (2/500 cilia), confirming that the PPC is a transient structure (Supplementary Fig. 8a). Smo-EGFP showed similar transient structures in mouse inner medullary collecting duct (IMCD) cells (Supplementary Fig. 8b).

Notably, the majority of newly formed cilia (13/17 cilia) displaying mature lengths $(4.5 \pm 1.9 \mu \mathrm{m})$ in ACTR3-depleted live cells maintained pronounced PPC at the ciliary base (Fig. 1i and Supplementary Fig. 7b), although the PPC eventually disappeared after $24 \mathrm{~h}$ serum starvation as in the control (Supplementary Fig. 8a). Moreover, facilitated ciliogenesis 

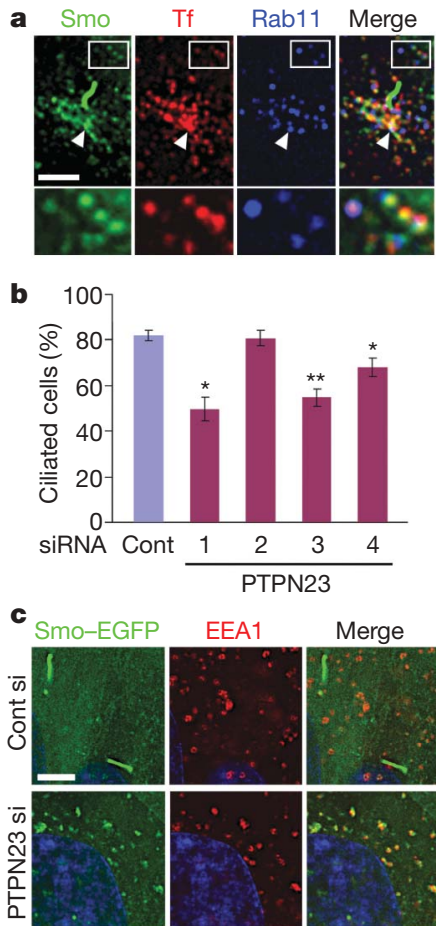

Merge

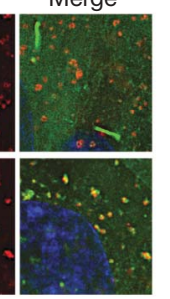

d PTPN23
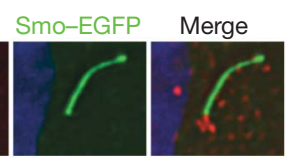

Figure 2 | Endocytic recycling pathway is linked to ciliogenesis. a, PPC overlapping with endocytosed transferrin-alexa 594 and Rab11

(arrowhead). b, PTPN23 knockdown decreased the number of ciliated cells. c, PTPN23 knockdown caused an accumulation of Smo-EGFP on EEA1positive early endosomes. d, PTPN23 immunofluorescence was detected at the ciliary base. e, PLA2G3 knockdown increased the number of cells with longer cilia $(>6 \mu \mathrm{m})$. f, PLA2G3 knockdown induced ciliogenesis without serum starvation. g, Pericentriolar localization of PLA2G3. h, PLA2G3 knockdown increased the number of cells exhibiting recycling endosomes concentrated at high levels around the centrosome (arrowheads). $\gamma$-Tub, $\gamma$-tubulin. Values, mean \pm s.d. $(n=4(\mathrm{~b}, \mathbf{e})$ and $3(\mathbf{f}))$. Student's $t$-test: ${ }^{*}$, $P<0.05$, **, $P<0.01$. Scale bars, $2.5 \mu \mathrm{m}$ (a, d, g); $5 \mu \mathrm{m}(\mathbf{c}) ; 20 \mu \mathrm{m}(\mathbf{h})$.

in cytochalasin D-treated cells was preceded by a promotion of PPC formation (16 PPC positive cells/30 live-imaged cells treated with cytochalasin D for $2 \mathrm{~h}$ versus 5 PPC-positive cells/30 control cells; Fig. 1l). Short-term live imaging showed that cytochalasin $\mathrm{D}$ also affects the stability of the pre-existing PPC (Supplementary Fig. 9). These observations indicate that actin network formation may negatively modulate ciliogenesis by destabilizing the PPC, a potential temporary reservoir of lipid and membrane proteins for efficient ciliogenesis.

Recycling endosomes are a dynamic vesiculotubular compartment, exporting endocytosed membrane proteins and lipids to the cell surface via vesicular intermediates ${ }^{18}$. We found that the PPC extensively overlaps with a subset of recycling endosomes concentrated around the centrosome: the PPC was labelled by endocytosed transferrin and recycling endosome marker Rab11 (Fig. 2a and Supplementary Fig. 10). PTPN23, a non-transmembrane tyrosine phosphatase, has been implicated in cargo sorting at early endocytic compartments ${ }^{19}$, and is one of the high-scored hits. Silencing of PTPN23 significantly reduced the number of ciliated cells (Fig. 2b and Supplementary Fig. 11). In addition, PTPN23 knockdown caused an accumulation of Smo-EGFP on the early endosomes marked by EEA1 (Fig. 2c). The PPC was not observed in the cells exhibiting early endosomal Smo-EGFP accumulation (0/80 cells; Supplementary Fig. 12). Supporting the link between recycling endosomes and ciliogenesis, knockdown of ASAP1 (not included in the initial screen library), a gene required for pericentrosomal enrichment of recycling endosomes ${ }^{20}$, caused a decrease in the number of ciliated cells (Supplementary Fig. 13a, b, c). These observations indicate that a trafficking pathway connecting endocytic compartments to the PPC is involved in cilium assembly. Pharmacological blocking of endocytic degradation pathway to lysosomes using concanamycin A did not block PPC formation or ciliogenesis, indicating that the endocytic recycling pathway is specifically involved in ciliogenesis (Supplementary Fig. 13d). Detection of PTPN23 immunoreactivity at the basal bodies further supported a direct role for PTPN23 in ciliary vesicle targeting (Fig. 2 d).

Among the unexpected screen hits was PLA2G3, a secreted phospholipase $^{21}$. Involvement of cytoplasmic phospholipase A2 enzymes in intracellular vesicle trafficking has been suggested on the basis of their potential for introducing membrane curvature ${ }^{22}$. Depletion of PLA2G3 facilitated cilium extension and induced ciliogenesis independently of serum starvation, indicating that PLA2G3 is a negative ciliogenesis regulator (Fig. 2e, f and Supplementary Fig. 14). Confirming this result, application of oleyloxyethyl phosphorylcholine (OPC), an inhibitor for secreted phospholipase ${ }^{23}$, increased the number of ciliated cells (Supplementary Fig. 15a). Notably, PLA2G3 immunofluorescence was detected at the centriole pair (Fig. $2 \mathrm{~g}$ ). In accord with its localization, knockdown of PLA2G3 significantly increased the number of cells displaying recycling endosomes concentrated at high levels around the centrosome $(17.4 \pm 1.4 \%$ in control cells and $48.2 \pm 2.7 \%$ in PLA2G3-depleted cells examined after $56 \mathrm{~h}$ transfection without serum starvation; Student's $t$-test, $P=0.006$; Fig. 2h). The majority of pericentrosomal recycling endosomes in PLA2G3-depleted cells (122/153) were associated with the PPC or cilia. Overexpressed PLA2G3 exhibited an extensive co-localization with endocytosed transferrin and inhibited ciliogenesis (Supplementary Fig. 14d-f). These results further demonstrate the link between endocytic recycling pathway and ciliogenesis. Moreover, higher intensities of Smo-EGFP fluorescence was observed in cilia from cells depleted of PLA2G3, demonstrating an inhibitory role for PLA2G3 in ciliary membrane protein targeting (Supplementary Fig. 15b, c).

The fact that both axoneme assembly and ciliary membrane biogenesis were facilitated by inhibition of actin polymerization

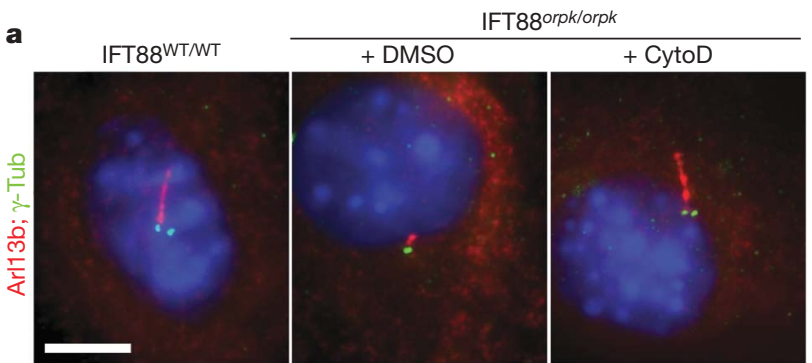

b

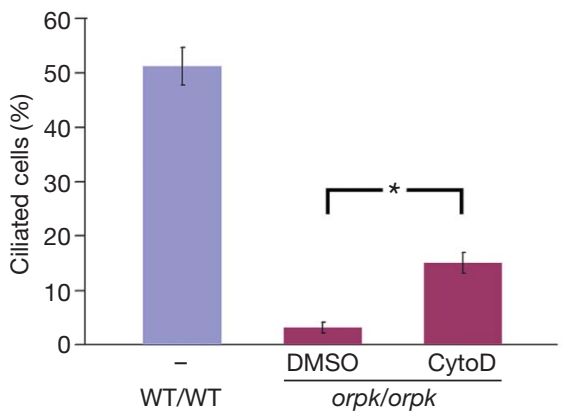

Figure 3 | Pharmacological rescue of ciliary defect on IFT88 mutant cells. a, Cytochalasin D treatment for $16 \mathrm{~h}$ partially rescued cilium elongation defect caused by hypomorphic IFT 88 mutation. $\mathbf{b}$, Percentage of cells with the primary cilium longer than $1.5 \mu \mathrm{m}$. WT, wild type.Values, mean \pm s.d. $(n=3)$. Student's $t$-test: ${ }^{*}, P<0.05$. Scale bar, $5 \mu \mathrm{m}$. 
(Supplementary Fig. 16) prompted us to test if cytochalasin D treatment could rescue ciliogenesis defect caused by loss-of-function mutation in core ciliogenesis machinery. IFT88 transports molecules required for cilium assembly ${ }^{4}$, and cells carrying homozygous IFT88 hypomorphic mutation (orpk/orpk) fail to grow cilia with normal length ${ }^{24}$. Notably, $16 \mathrm{~h}$ cytochalasin D treatment partially, but significantly, rescued ciliogenesis defect of IFT88 mutant cells (Fig. 3). A recent study showed that IFT particles are associated with recycling endosomes and are implicated in the recycling of T-cell receptors in lymphocytes $^{25}$. Thus it is likely that cytochalasin D facilitates ciliary recruitment of IFT particles through the endocytic recycling pathway. Cytochalasin D can interfere with many cellular processes involving actin polymerization, and thus is unlikely to be useful for ciliopathy treatment. However, small molecules specifically targeting actin modulators implicated in ciliogenesis might be good candidates for ciliopathy treatment.

In summary, our high-throughput functional screen identified modulators of ciliogenesis with diverse molecular functions, connecting ciliogenesis with other basic cellular processes. Furthermore, we discovered several proteins involved in the control of cilium length, a key determinant of normal cilium function. We anticipate that the development of small molecules that target these proteins will provide novel strategies for the treatment of the ciliopathies.

\section{METHODS SUMMARY}

Cell culture. htRPE cells were maintained in DMEM:F12 (Dulbecco's modified Eagle medium) supplemented with $10 \%$ fetal bovine serum. Plasmid DNA harbouring mouse Smo-EGFP fusion gene was transfected to htRPE cells and a stable cell line (htRPE-Smo-EGFP) was established by G418 selection. For ciliogenesis induction, culture medium was replaced with DMEM (without supplement) when cells are $\sim 90 \%$ confluent, and cultured for $48 \mathrm{~h}$ before fixation. Overexpressed Smo-EGFP was targeted to ciliary membrane independent of Sonic hedgehog.

siRNA library screen. An arrayed library containing 31,111 unique siRNAs targeting 7,784 human genes (Ambion; human druggable genome siRNA library V3.1; including siRNAs targeting 665 kinases, 101 kinase modulators, 231 phosphatases, 307 ligases, 57 lipases, 444 signalling molecules and 426 cytoskeletoninteracting proteins) was screened in duplicates. High-throughput imaging was done using an IC100 automated microscope system (Beckman Coulter), and the images were analysed by CytoShop software. For a confirmation screen, re-arrayed 913 siRNAs targeting 232 primary screen hits were tested in quadruplicates.

Full Methods and any associated references are available in the online version of the paper at www.nature.com/nature.

\section{Received 10 November 2009; accepted 4 February 2010.}

1. Gerdes, J. M., Davis, E. E. \& Katsanis, N. The vertebrate primary cilium in development, homeostasis, and disease. Cell 137, 32-45 (2009).

2. Eggenschwiler, J. T. \& Anderson, K. V. Cilia and developmental signaling. Annu. Rev. Cell Dev. Biol. 23, 345-373 (2007).

3. Fliegauf, M., Benzing, T. \& Omran, H. When cilia go bad: cilia defects and ciliopathies. Nature Rev. Mol. Cell Biol. 8, 880-893 (2007).

4. Rosenbaum, J. L. \& Witman, G. B. Intraflagellar transport. Nature Rev. Mol. Cell Biol. 3, 813-825 (2002).

5. Christensen, S. T. et al. The primary cilium coordinates signaling pathways in cell cycle control and migration during development and tissue repair. Curr. Top. Dev. Biol. 85, 261-301 (2008).

6. Inglis, P. N., Boroevich, K. A. \& Leroux, M. R. Piecing together a ciliome. Trends Genet. 22, 491-500 (2006).

7. Gherman, A., Davis, E. E. \& Katsanis, N. The ciliary proteome database: an integrated community resource for the genetic and functional dissection of cilia. Nature Genet. 38, 961-962 (2006)
8. Corbit, K. C. et al. Vertebrate Smoothened functions at the primary cilium. Nature 437, 1018-1021 (2005)

9. Bielas, S. L. et al. Mutations in INPP5E, encoding inositol polyphosphate-5phosphatase $\mathrm{E}$, link phosphatidyl inositol signaling to the ciliopathies. Nature Genet. 41, 1032-1036 (2009).

10. Fernandez-Gonzalez, A. et al. Purkinje cell degeneration ( $p c d$ ) phenotypes caused by mutations in the axotomy-induced gene, Nna1. Science 295, 1904-1906 (2002).

11. Silacci, P. et al. Gelsolin superfamily proteins: key regulators of cellular functions. Cell. Mol. Life Sci. 61, 2614-2623 (2004).

12. Cooper, J. A. \& Schafer, D. A. Control of actin assembly and disassembly at filament ends. Curr. Opin. Cell Biol. 12, 97-103 (2000).

13. Sepulveda, J. L. \& Wu, C. The parvins. Cell. Mol. Life Sci. 63, 25-35 (2006).

14. Sorokin, S. Centrioles and the formation of rudimentary cilia by fibroblasts and smooth muscle cells. J. Cell Biol. 15, 363-377 (1962).

15. Archer, F. L. \& Wheatley, D. N. Cilia in cell-cultured fibroblasts. II. Incidence in mitotic and post-mitotic BHK 21-C13 fibroblasts. J. Anat. 109, 277-292 (1971).

16. Nachury, M. V. et al. A core complex of BBS proteins cooperates with the GTPase Rab8 to promote ciliary membrane biogenesis. Cell 129, 1201-1213 (2007).

17. Yoshimura, S. et al. Functional dissection of Rab GTPases involved in primary cilium formation. J. Cell Biol. 178, 363-369 (2007).

18. Maxfield, F. R. \& McGraw, T. E. Endocytic recycling. Nature Rev. Mol. Cell Biol. 5, 121-132 (2004).

19. Doyotte, A., Mironov, A., McKenzie, E. \& Woodman, P. The Bro1-related protein HD-PTP/PTPN23 is required for endosomal cargo sorting and multivesicular body morphogenesis. Proc. Natl Acad. Sci. USA 105, 6308-6313 (2008).

20. Inoue, H., Ha, V. L., Prekeris, R. \& Randazzo, P. A. Arf GTPase-activating protein ASAP1 interacts with Rab11 effector FIP3 and regulates pericentrosomal localization of transferrin receptor-positive recycling endosome. Mol. Biol. Cell 19, 4224-4237 (2008).

21. Valentin, E. et al. Novel human secreted phospholipase A(2) with homology to the group III bee venom enzyme. J. Biol. Chem. 275, 7492-7496 (2000).

22. Brown, W. J., Chambers, K. \& Doody, A. Phospholipase A2 (PLA2) enzymes in membrane trafficking: mediators of membrane shape and function. Traffic 4, 214-221 (2003).

23. Hope, W. C., Chen, T. \& Morgan, D. W. Secretory phospholipase A2 inhibitors and calmodulin antagonists as inhibitors of cytosolic phospholipase A2. Agents Actions 39 (Spec No), C39-C42 (1993).

24. Yoder, B. K. et al. Polaris, a protein disrupted in orpk mutant mice, is required for assembly of renal cilium. Am. J. Physiol. Renal Physiol. 282, F541-F552 (2002).

25. Finetti, F. et al. Intraflagellar transport is required for polarized recycling of the TCR/CD3 complex to the immune synapse. Nature Cell Biol. 11, 1332-1339 (2009).

Supplementary Information is linked to the online version of the paper at www.nature.com/nature.

Acknowledgements The screen was performed at the Functional Genomics Core (LINCC grant P30 NS057096 to S. Lipton) with technical assistance from A. Cortez and E. Sisman and with imaging support from the UCSD Microscopy Core (P30 NS047101 and P30 CA23100). We thank P. A. Beachy for the Smo-EGFP plasmid and J. F. Reiter for IFT88 orpk/orpk MEFs. We thank C. Kintner, V. M. Fowler, S. J. Field and S. Ferro-Novick for discussion. This work was supported by grants from NARSAD (Young Investigator Award to J.K.), NINDS (RO1 NS052455 to

J.G.G.), the NIH (GM070743 to T.I. and K.L.), the Korea Food and Drug

Administration (10182KFDA992 to K.L.) and the Brain Research Center of the 21st Century Frontier Research Program (M103KV010008-07K2201-00810 to K.L.). J.G.G. is an investigator of the Howard Hughes Medical Institute.

Author Contributions J.G.G. conceived and directed the project. J.K., S.H.-G. and P.A.-B. designed the screen and J.K., S.H.-G. and E.S. performed the screen. J.K., K.O., K.L. and T.I. analysed the screen data. J.K. designed the follow-up experiments and J.K. and J.E.L. performed the experiments. J.K. interpreted the results and J.K. and J.G.G. wrote the paper with contributions from S.H.-G. and P.A.-B.

Author Information Reprints and permissions information is available at www.nature.com/reprints. The authors declare competing financial interests: details accompany the full-text HTML version of the paper at www.nature.com/ nature. Correspondence and requests for materials should be addressed to J.G.G. (jogleeson@ucsd.edu). 


\section{METHODS}

Primary screen. Assay plates (384-well plate with optical bottom; Greiner) were spotted with $1 \mu \mathrm{l}$ of $0.5 \mu \mathrm{M}$ siRNA using the Valocity 11-Bravo Pipettor with a 384 ST head. To acquire high-quality primary screen data, four unique siRNAs were typically used to knockdown each gene and all siRNAs were tested individually in duplicate. KIF3A siRNAs were included in all the plates as a positive control. Reverse transfection was performed using Lipofectamine RNAiMAX: final siRNA concentration was $10 \mathrm{nM}$. htRPE-Smo-EGFP cells were suspended in DMEM:F12 supplemented with 10\% FBS, and seeded onto assay plates using the Matrix-Well Mate (4,000 cells in $40 \mu \mathrm{l}$ medium for each well). Culture medium was replaced with DMEM $24 \mathrm{~h}$ after transfection using the TiterTekMAP-C, and cells were incubated for additional $48 \mathrm{~h}$ before fixation.

Imaging and image analysis. Image acquisition of the siRNA screen was performed on the IC100 imaging system (Beckman Coulter) equipped with an ORCA-ER scientific camera (Hamamatsu) and a multi-plate handling BRT system (Beckman Coulter). Images were acquired using a Nikon Plan Fluor $\times 200.5$ NA objective. Six fields were acquired throughout each well at two wavelengths: nuclear DAPI and GFP. CytoShop HCS analysis software (Beckman Coulter) was used to perform image segmentation and cytometry. The nucleus of each cell was identified from the DAPI images using CytoShop's 'Nuclear Segmentation' algorithm that included an 'open' morphological operation to separate touching nuclei. Equidistance tessellation lines were drawn between the centroids of the identified nuclei, effectively breaking up the images into cellular regions. Cilia detection was performed on the GFP images using CytoShop's 'GPCR Segmentation' algorithm. An Object Extraction Correlation radius of 30 pixels was set, inside which pixels were assumed to belong to the cytoplasm of the cell. The object scale for the aggregate (cilia) detection was iteratively adjusted to 8 pixels (average object $=$ cilia size) and the minimum intensity peak height was set to 15 (minimum difference in intensity between cilia object and neighbouring image intensities). The number of detected aggregates (cilia) was then reported for each cell object and used to determine the percentage of cells per well that were cilia positive. Plate-to-plate variability was normalized by a control-based method: Normalized percent inhibition $(\mathrm{NPI})=($ mean of negative control $-\mathrm{Xi}) /($ mean of negative control-mean of positive control) $\times 100$, where $X \mathrm{i}$ is the means of ciliated cell count (\%) for each siRNA, negative control is mock transfection and positive control is KIF3A siRNA ( $n=4$ per plate). Threshold for selecting active siRNAs was \pm 1.5 s.d. from the overall mean. Genes were defined as primary screen hits if at least two independent siRNAs showed NPI values above the threshold. Data from toxic siRNAs that decreased cell count by $2 \geq$ s.d. from overall mean were excluded, and additional invalid data ascribed to cell toxicity or out-of-focus images were excluded through manual inspection.

Confirmation screen. For a confirmation screen, htRPE-Smo-EGFP cells (6,500 cells per well) were transfected and serum-starved as in the primary screen. The confirmation screen was based on measurement of (1) the percentage of ciliated cells for positive modulators and (2) mean cilium size (pixel sum, arbitrary unit) for negative modulators. For plate normalization, quantification data was converted to $Z$ scores using 48 unique non-targeting siRNAs included to each plate as references: $Z$ score $=(X \mathrm{i}$-median of 48 control siRNAs $) /$ $1.4826 \times$ MAD of 48 control siRNAs, where $X i$ is quantification data and MAD is median absolute deviation. Genes were defined as confirmation screen hits if at least two siRNAs showed $Z$ scores $\leq-2$ (for positive modulators) or $\geq 1.15$ (for negative modulators). Target sequences for selected siRNAs used for the follow-up studies are given in Supplementary Table 4.
Hit classification. To identify genes required for Smo-EGFP targeting to cilia without affecting ciliogenesis, the confirmed positive modulators were rescreened: htRPE cells were transfected with siRNAs (two best-scored siRNAs for each gene), serum-starved as above and examined by anti-acetylated-tubulin immunofluorescence staining (Supplementary Table 3). To classify further the confirmed negative modulators, htRPE-Smo-EGFP cells were transfected with siRNAs (two best scored siRNAs for each gene) for 3 days without serum starvation (Supplementary Table 3).

Immunofluorescence and western blotting. For indirect immunofluorescence, cells were fixed in either methanol or $4 \%$ paraformaldehyde (PFA) depending on antigen. Cells for anti-acetylated tubulin staining were incubated on ice for $45 \mathrm{~min}$ before fixation to destroy cytoskeletal microtubules. Primary antibodies used for immunofluorescence are: mouse anti-acetylated-tubulin (Sigma), rabbit antiGlu-tub (Chemicon), mouse anti-EEA1 (BD Transduction Laboratory), mouse anti-Rab11 (BD Transduction Laboratory), mouse anti-PTPN23 (Abnova), mouse anti-PLA2G3 (Abnova), rabbit-anti-Arl13b (a gift from T. Caspary), and rabbit anti-gamma-tubulin (Sigma). Alexa 488- or Alexa 594-conjugated secondary antibodies (Molecular Probes) were applied for $1 \mathrm{~h}$ at room temperature. For immunoblotting, cells were extracted with RIPA lysis buffer 2.5 days after transfection and boiled with SDS sample buffer. Same volumes of lysates were loaded for paired experiments. Primary antibodies used for western blotting are: mouse anti-GSN (Genetex), rabbit anti-ACTR3 (Sigma), rabbit anti-PARVA (Cell Signaling), mouse anti-PTPN23 (Abnova) and mouse anti-PLA2G3 (Abnova). Bound antibodies were detected using horseradish peroxidase-conjugated secondary antibodies (Pierce). Membranes were stripped and re-blotted with anti-alpha-tubulin antibody (Sigma).

Live cell imaging. htRPE-Smo-EGFP cells were transfected with siRNAs for 2.5 days in the presence of $10 \%$ serum and then non-ciliated cells were liveimaged for $6 \mathrm{~h}$ in serum-free medium (Fig. 1i). Images $(2 \mu \mathrm{m} \mathrm{Z}$-stack) were acquired using an Olympus IX70 microscope and a cooled charge-coupled device system with temperature and $\mathrm{CO}_{2}$ control.

Internalization of transferrin. Cells were incubated with iron-saturated human transferrin-AlexaFluor594 (30 $\mathrm{g} \mathrm{ml}^{-1}$; Invitrogen) in serum-free medium for $45 \mathrm{~min}$ at $37^{\circ} \mathrm{C}$. Cells were rinsed quickly three times with ice-cold DMEM containing $10 \%$ FBS and incubated for $5 \mathrm{~min}$ at $37^{\circ} \mathrm{C}$ to clear transferrinalexaFluor 594 from the plasma membrane and early endosomes. Cells were fixed with $4 \%$ PFA on ice.

Pharmacological studies. Cytochalasin D (Sigma) resuspended in DMSO was applied to cells at various conditions (see text and figure legends). Concanamycin A (Sigma) resuspended in DMSO was applied to cells at $2 \mu \mathrm{M}$ in the absence of serum for final $16 \mathrm{~h}$ before fixation. OPC (BIOMOL) resuspended in ethanol was applied to cell at $25 \mu \mathrm{M}$ for final $36 \mathrm{~h}$ before fixation in the presence of $10 \%$ serum (OPC was cytotoxic when treated at $25 \mu \mathrm{M}$ in the absence of serum). For a vehicle control, an equivalent amount of DMSO or ethanol was added to cells.

Fluorescence intensity measurement. Exposure was set to minimum and EGFP fluorescence was imaged using an Olympus IX70 microscope and a cooled charge-coupled device system. Small areas including cilium were excised from the images and placed in a panel using Adobe Photoshop 7.0 software. Maximum pixel intensity of EGFP fluorescence from each cilium was measured using ImageJ software.

Cell counting and statistics. The total numbers of cells counted are: $>2,000$ (Figs $1 \mathrm{~b}$ and 2b), $>500$ (Figs $1 \mathrm{~d}, \mathrm{f}$ and $2 \mathrm{e}, \mathrm{f}$ ) and $>300$ (Figs $1 \mathrm{~g}, \mathrm{~h}$ and $3 \mathrm{~b}$ ). Binucleated cells were excluded from the counting for Fig. 3b. Data from followup studies were analysed by two-tailed, paired Student's $t$-test using $t$-test calculation software (GraphPad). 